\title{
The Hausdorff dimension of the projections of self-affine carpets
}

\author{
by \\ Andrew Ferguson (Coventry), Thomas Jordan (Bristol) \\ and Pablo Shmerkin (Manchester)
}

\begin{abstract}
We study the orthogonal projections of a large class of self-affine carpets, which contains the carpets of Bedford and McMullen as special cases. Our main result is that if $\Lambda$ is such a carpet, and certain natural irrationality conditions hold, then every orthogonal projection of $\Lambda$ in a non-principal direction has Hausdorff dimension $\min (\gamma, 1)$, where $\gamma$ is the Hausdorff dimension of $\Lambda$. This generalizes a recent result of Peres and Shmerkin on sums of Cantor sets.
\end{abstract}

1. Introduction and statement of results. A basic problem in fractal geometry is to understand how the Hausdorff dimension of a set behaves under orthogonal projections. Results which are valid for almost every projection are well-known, going back to the celebrated Marstrand Projection Theorem [8]: let $\Lambda \subset \mathbb{R}^{2}$ be a Borel set, and denote by $\operatorname{proj}_{\theta}$ the orthogonal projection onto a line making angle $\theta$ with the origin. Then

$$
\operatorname{dim}_{\mathrm{H}}\left(\operatorname{proj}_{\theta}(\Lambda)\right)=\min \left(\operatorname{dim}_{\mathrm{H}}(\Lambda), 1\right) \quad \text { for almost every } \theta,
$$

where $\operatorname{dim}_{\mathrm{H}}$ stands for Hausdorff dimension. See [9, Chapter 9], [13] and references therein for many extensions of Marstrand's Theorem. We underline that in all cases, the proof is non-constructive and gives no indication of what the exceptional set of directions may look like (other than giving a bound on its dimension). This motivates the following general question: if $\Lambda \subset \mathbb{R}^{2}$ is dynamically defined, is it possible to determine the set of exceptional directions in Marstrand's Theorem explicitly?

Recall that the one-dimensional Sierpiński gasket $\mathcal{S}$ is defined as

$$
\mathcal{S}=\left\{\sum_{i=1}^{\infty} 3^{-i} a_{i}: a_{i} \in\{(0,0),(1,0),(0,1)\}\right\} .
$$

2010 Mathematics Subject Classification: Primary 28A80, 28A78.

Key words and phrases: Hausdorff dimension, orthogonal projection, self-affine carpet. 
A question, attributed to Furstenberg, is whether $\operatorname{proj}_{\theta}(\mathcal{S})$ has Hausdorff dimension one for all $\theta$ with irrational slope; this problem remains open. On the other hand, Moreira (see [11]) succeeded in showing that for the cartesian product of certain non-linear dynamically defined Cantor sets on the real line, there are no exceptional directions, other than $\theta=0, \pi / 2$ which are trivial ones (his motivation was to find the dimension of the arithmetic sum of two such Cantor sets; note that the arithmetic sum $A+B$ is affinely equivalent to a projection $\left.\operatorname{proj}_{\pi / 4}(A \times B)\right)$. Peres and Shmerkin [14] obtained an analogous result for products of linear self-similar sets and for self-similar sets in the plane. The main motivating class of examples in their work are the product sets $\Lambda=C_{a} \times C_{b}$, where $C_{s}$ is the central Cantor set which is obtained by replacing the unit interval $[0,1]$ by the union $[0, s] \cup[1-s, 1]$ and iterating. All these positive results require an appropriate irrationality assumption; for $\Lambda=C_{a} \times C_{b}$ this reduces to $\log b / \log a$ being an irrational number. Very recently, Hochman and Shmerkin [5] introduced a general approach that unifies and extends all these results.

In the present work we continue this line of research. We focus on a family of dynamically defined fractals generally known as self-affine carpets. These carpets are defined by replacing the unit square $Q$ with a union of pairwise non-overlapping rectangles $\left\{S_{i}(Q)\right\}$ satisfying some geometric arrangement, and iterating inside each $S_{i}(Q)$; here the $S_{i}$ are affine maps with a diagonal linear part. A simple model of self-affine carpets was introduced by Bedford [2] and McMullen [10, who independently found a formula for their Hausdorff dimension. Figure 1 shows a typical Bedford-McMullen carpet. We will be concerned with two more general classes of self-affine carpets, the class studied by Gatzouras and Lalley [7], and the class recently introduced by Barański [1]; precise definitions are given below. Our main result is that if $\Lambda$ is either a Gatzouras-Lalley or a Barański carpet, and a natural irrationality condition holds, then

$$
\operatorname{dim}_{H}\left(\operatorname{proj}_{\theta}(\Lambda)\right)=\min \left(\operatorname{dim}_{H}(\Lambda), 1\right) \quad \text { for all } \theta \in(0, \pi) \backslash\{\pi / 2\} .
$$

(See Theorem 1.1 below for the precise statement.) In other words, for these carpets the only possible exceptional directions in Marstrand's Theorem are 0 and $\pi / 2$; it will be clear from the definitions that these directions can indeed be exceptional. We underline that the aforementioned results in [11], [14, 5] all rely on the conformality of the underlying constructions. The self-affine carpets we study are intrinsically non-conformal.

Recall that an iterated function system on $\mathbb{R}^{d}$ is a finite collection $\left\{S_{1}\right.$, $\left.\ldots, S_{k}\right\}$ of maps from $\mathbb{R}^{d}$ into itself which are strictly contractive, i.e. there exists $0<c<1$ such that $\left|S_{i}(x)-S_{i}(y)\right|<c|x-y|$ for all $x, y \in \mathbb{R}^{d}$ and all $i$. It is well-known that there exists a unique non-empty compact set $\Lambda$, called the attractor or invariant set of the iterated function system, such 

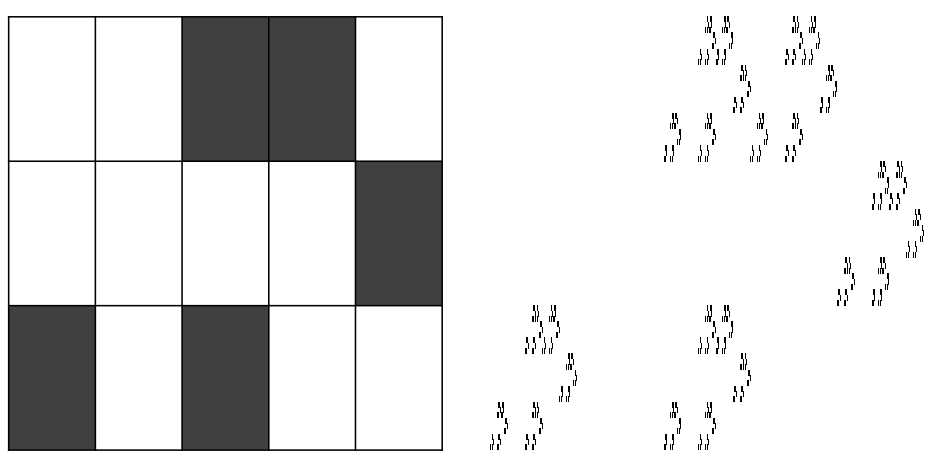

Fig. 1. A generating pattern of a Bedford-McMullen carpet (left) and the associated invariant set $\Lambda$ (right)

that $\Lambda=\bigcup_{i} S_{i}(\Lambda)$. In the case in which all the $S_{i}$ are homotheties, $\Lambda$ is called a self-similar set. We will be concerned with the case in which all the $S_{i}$ are affine maps, in which case $\Lambda$ is known as a self-affine set. The reader is referred to [4] for further background on iterated function systems and self-similar sets.

One reason why self-affine carpets are of interest is that they are among the simplest planar constructions which are not self-similar. They exhibit phenomena not present in the self-similar setting, like the non-coincidence of Hausdorff and packing dimension (see [7]) or the infinitude of Hausdorff measure in the critical dimension [12]. Our results suggest that from the point of view of orthogonal projections, these carpets behave rather like products of self-similar sets; compare Theorem 1.1 with [14, Theorem 5].

Let us give the precise definitions of the constructions we will consider. The first type was introduced by Gatzouras and Lalley in [7]. For this construction we fix positive integers $m, n_{1}, \ldots, n_{m}$ and set $D=\{(i, j): 1 \leq i$ $\left.\leq m, 1 \leq j \leq n_{i}\right\}$. Let $\Lambda$ be the unique non-empty compact set satisfying

$$
\Lambda=\bigcup_{(i, j) \in D} S_{i j}(\Lambda)
$$

where the map $S_{i j}$ is of the form

$$
S_{i j}(x, y)=\left(a_{i j} x+c_{i j}, b_{i} y+d_{i}\right) .
$$

We impose the following conditions on the maps $S_{i j}: 0<a_{i j}<b_{i}<1$ for each pair $(i, j)$, and the intervals $\left\{b_{i} I+d_{i}\right\}_{i=1}^{m},\left\{a_{i j} I+c_{i j}\right\}_{j=1}^{n_{i}}$ have disjoint interiors, where $I=[0,1]$ is the unit interval; see Figure 2. For any $n \in \mathbb{N}$, we will let

$$
\mathbb{P}^{n}=\left\{\left(p_{1}, \ldots, p_{n}\right): p_{i} \geq 0 \text { and } \sum_{i=1}^{n} p_{i}=1\right\}
$$


be the space of all probability vectors with $n$ elements. It is proven in [7] (albeit with a slightly different formulation) that the Hausdorff dimension of $\Lambda$ is given by

$$
\operatorname{dim}_{\mathrm{H}}(\Lambda)=\sup _{\mathbf{p} \in \mathbb{P}^{m}}\left\{\frac{\sum_{i=1}^{m} p_{i} \log p_{i}}{\sum_{i=1}^{m} p_{i} \log b_{i}}+t(\mathbf{p})\right\}
$$

where $t(\mathbf{p})$ is the unique real number satisfying

$$
\sum_{i=1}^{m} p_{i} \log \left(\sum_{j=1}^{n_{i}} a_{i j}^{t(\mathbf{p})}\right)=0 .
$$

We will say a Gatzouras-Lalley construction is of irrational type if either

(1) there exists $(i, j) \in D$ with $\log a_{i j} / \log b_{i} \notin \mathbb{Q}$, or

(2) there exist $i, j, k$ with $\log a_{i j} / \log b_{k} \notin \mathbb{Q}$ and $\log a_{i j} / \log b_{i}$ is not constant for all $i, j$.

A special case of the Gatzouras-Lalley construction is the situation where $n_{1}=\cdots=n_{m}$ and there are constants $0<a<b<1$ such that each $a_{i j}=a$ and $b_{i}=b$. We will refer to this as the homogeneous uniform fibre case.
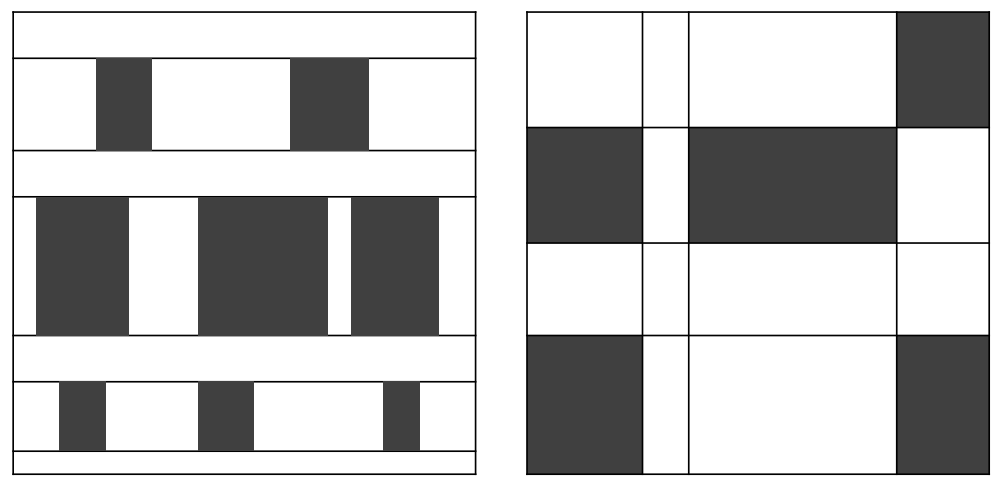

Fig. 2. Generating patterns for a Gatzouras-Lalley carpet (left) and a Barański carpet (right)

An alternative construction was considered by Barański in [1]. For this construction fix positive integers $m, n$ and let $D \subseteq\{(i, j): 1 \leq i \leq n$ and $1 \leq j \leq m\}$. For each $1 \leq i \leq n$ and $1 \leq j \leq m$ we fix values $0<a_{i}, b_{j}<1$ such that $\sum_{i=1}^{n} a_{i}=\sum_{j=1}^{m} b_{j}=1$. We then consider the affine iterated function system defined by the maps

$$
S_{i j}(x, y)=\left(a_{i} x+\sum_{l=1}^{i-1} a_{l}, b_{j} y+\sum_{l=1}^{j-1} b_{l}\right)
$$


for each $(i, j) \in D$. Again we let $\Lambda$ be the unique non-empty compact set satisfying

$$
\Lambda=\bigcup_{(i, j) \in D} S_{i j}(\Lambda)
$$

(See Figure 2,) We remark that Bedford-McMullen carpets are the special case of the Barański construction in which $a_{i}=1 / n$ and $b_{j}=1 / m$ for all $i, j$.

In [1] Barański computed the dimension of such attractors. To state his result we denote the elements of a probability vector $\mathbf{p} \in \mathbb{P}^{|D|}$ by $p_{i j}$ where $|D|$ denotes the cardinality of $D$. For $1 \leq i \leq n$ let $q_{i}(\mathbf{p})=\sum_{j:(i, j) \in D} p_{i j}$ and for $1 \leq j \leq m$ let $r_{j}(\mathbf{p})=\sum_{i:(i, j) \in D} p_{i j}$. We now define

$$
\begin{aligned}
& d_{x}=\sup _{\mathbf{p} \in \mathbb{P}^{|D|}}\left\{\frac{\sum_{i=1}^{n} q_{i}(\mathbf{p}) \log q_{i}(\mathbf{p})}{\sum_{i=1}^{n} q_{i}(\mathbf{p}) \log a_{i}}+\frac{\sum_{i j} p_{i j} \log \left(p_{i j} / q_{i}(\mathbf{p})\right)}{\sum_{j=1}^{m} r_{j}(\mathbf{p}) \log b_{j}}\right\}, \\
& d_{y}=\sup _{\mathbf{p} \in \mathbb{P}^{|D|}}\left\{\frac{\sum_{j=1}^{m} r_{j}(\mathbf{p}) \log r_{j}(\mathbf{p})}{\sum_{j=1}^{m} r_{j}(\mathbf{p}) \log b_{j}}+\frac{\sum_{i j} p_{i j} \log \left(p_{i j} / r_{j}(\mathbf{p})\right)}{\sum_{i=1}^{n} q_{i}(\mathbf{p}) \log a_{i}}\right\} .
\end{aligned}
$$

Barański showed that

$$
\operatorname{dim}_{H}(\Lambda)=\max \left\{d_{x}, d_{y}\right\} .
$$

We will call the system of irrational type if either

(1) we can find $(i, j) \in D$ such that $\log a_{i} / \log b_{j} \notin \mathbb{Q}$, or

(2) we can find $(i, j) \notin D$ such that $\log a_{i} / \log b_{j} \notin \mathbb{Q}$ and there exist $(i, j),(k, l) \in D$ with $\log a_{i} / \log b_{j} \neq \log a_{k} / \log b_{l}$.

We now state our main result:

THEOREM 1.1. Let $\Lambda$ be the attractor for an iterated function system defined by the Gatzouras-Lalley construction or the Barański construction. If the system is of irrational type, then

$$
\operatorname{dim}_{\mathrm{H}}\left(\operatorname{proj}_{\theta}(\Lambda)\right)=\min \left(\operatorname{dim}_{\mathrm{H}}(\Lambda), 1\right)
$$

for all $\theta \in(0, \pi) \backslash\{\pi / 2\}$.

The Barański class contains the products $C_{a} \times C_{b}$ as special cases, and also the cartesian products of more general self-similar sets in the line; hence, Theorem 1.1 generalizes [14, Theorem 2]. We underline that the proofs in [14] rely crucially on the product structure of $\Lambda=C_{a} \times C_{b}$. More precisely, in that paper the following fact is key: $\Lambda$ can be written as a union $\bigcup_{i} \Lambda_{i}$, where each $\Lambda_{i}$ is the image of $\Lambda$ under an affine map of small distortion. This structure breaks down already for Bedford-McMullen carpets such as the one depicted in Figure 1. Thus, although we follow the general pattern of proof of [14, Theorem 2], we must also introduce new ideas to deal with the lack of product structure. 
A key to the proof of Theorem 1.1 is to first prove the homogeneous uniform fibre Gatzouras-Lalley case, and then use probabilistic ideas to deduce the general case.

Proposition 1.2. Let $\Lambda$ be an attractor for a homogeneous uniform fibre Gatzouras-Lalley construction. If $\log a / \log b \notin \mathbb{Q}$ then

$$
\operatorname{dim}_{H}\left(\operatorname{proj}_{\theta}(\Lambda)\right)=\min \left(\operatorname{dim}_{\mathrm{H}}(\Lambda), 1\right)
$$

for all $\theta \in(0, \pi) \backslash\{\pi / 2\}$.

In [6], Kenyon and Peres studied a generalization of Bedford-McMullen carpets which are modelled by sofic symbolic systems (recall that a sofic system is a factor of a subshift of finite type on a finite alphabet). We can describe their construction as follows: Fix integers $2 \leq m<n$. Let $X$ be a closed subset of the symbolic space $D^{\mathbb{N}}$, where $D=\{1, \ldots, n\} \times\{1, \ldots, m\}$, and assume $X$ is $T$-invariant and sofic, where $T$ is the shift map on $D^{\mathbb{N}}$. For $(i, j) \in D$, write

$$
S_{(i, j)}(x, y)=((x+i) / n,(y+j) / m) .
$$

Further, set

$$
\Lambda=\Lambda(X)=\bigcap_{\ell=1}^{\infty} \bigcup_{|\sigma|=\ell, \sigma \in X^{*}} S_{\sigma_{1}} \circ \cdots \circ S_{\sigma_{\ell}}([0,1] \times[0,1]),
$$

where $X^{*}$ is the set of all finite strings which can be continued to an infinite string in $X$. It is easy to see that in the case $X=\left(D^{\prime}\right)^{\mathbb{N}}$, where $D^{\prime} \subset D$, the set $\Lambda$ is a Bedford-McMullen carpet, and thus a Barański construction, and is of irrational type whenever $\log m / \log n \notin \mathbb{Q}$.

Kenyon and Peres obtained a formula for the Hausdorff dimension of such sofic affine-invariant sets in terms of some random matrix products [6. Theorems 1.1 and 3.2]. Of importance to us is that in the course of the proof of their Theorem 3.2, Kenyon and Peres construct Bedford-McMullen carpets $\Lambda_{j}$ corresponding to a subset $D_{j} \subset\left\{0, \ldots, n^{j}\right\} \times\left\{0, \ldots, m^{j}\right\}$, such that $\Lambda_{j} \subset \Lambda$ for all $j$, and $\operatorname{dim}_{\mathrm{H}}\left(\Lambda_{j}\right) \rightarrow \operatorname{dim}_{\mathrm{H}}(\Lambda)$ as $j \rightarrow \infty$. Hence we have the following immediate corollary of Theorem 1.1 .

Corollary 1.3. Suppose that $\log m / \log n$ is irrational and $\Lambda$ is defined by (1.2) for some sofic system $X$. Then

$$
\operatorname{dim}_{\mathrm{H}}\left(\operatorname{proj}_{\theta}(\Lambda)\right)=\min \left(\operatorname{dim}_{\mathrm{H}}(\Lambda), 1\right)
$$

for all $\theta \in(0, \pi) \backslash\{\pi / 2\}$.

When seen as a subset of the torus $T^{2}$, the set $\Lambda$ is invariant under the expanding toral endomorphism $A(x, y)=(m x, n y)(\bmod 1)$. We do not know if the above corollary remains true if $\Lambda$ is an arbitrary closed $A$-invariant 
subset of the torus (this would correspond to $X$ being an arbitrary subshift, not necessarily sofic).

Section 2 contains a discrete projection theorem, while Section 3 contains the proof of Proposition 1.2. In Section 4 we then use probabilistic ideas and Proposition 1.2 to complete the proof of Theorem 1.1. Throughout the article, $\mathcal{L}$ will be used to denote Lebesgue measure.

2. A discrete projection theorem. The main idea behind the proof of Proposition 1.2 is that for a self-affine carpet $\Lambda$, and a fixed angle $\theta \in$ $(0, \pi / 2)$, we can construct a subset $\Lambda_{\theta} \subset \Lambda$ with the following properties: $\Lambda_{\theta}$ may be written as an intersection over a nested family of compact sets, i.e. $\Lambda_{\theta}=\bigcap_{k} \bigcup_{Q \in \mathcal{Q}_{k}} Q$, such that for each $k$, the elements of the family $\left\{\operatorname{proj}_{\theta}(Q): Q \in \mathcal{Q}_{k}\right\}$ are suitably separated. The following theorem gives sufficient conditions for the cardinality of the sets $\mathcal{Q}_{k}$ being large enough so that the resulting set $\Lambda_{\theta}$ has dimension that is arbitrarily close to that of $\Lambda$. It plays a similar role to that of [14, Proposition 7], and is a variation on this result.

Theorem 2.1. Given constants $A>1, A_{1}, A_{2}>0$ and $\gamma \in(0,1)$, there exists a constant $\delta$ such that the following holds: Fix $\rho>0$. Let $\mathcal{Q}$ be a collection of convex subsets of the unit disk such that each element contains a disk of radius $A^{-1} \rho$ and is contained in a disk of radius $A \rho$, and any $Q, Q^{\prime} \in \mathcal{Q}$ with $Q \neq Q^{\prime}$ have disjoint interiors. Furthermore suppose that $\mathcal{Q}$ has cardinality at least $\rho^{-\gamma} / A_{1}$, yet any disk of radius $\ell \in(\rho, 1)$ intersects at most $A_{2}(\ell / \rho)^{\gamma}$ elements of $\mathcal{Q}$. Then for any $\varepsilon>0$ there exists a set $J \subset[0, \pi)$ with the following properties:

(i) $\mathcal{L}([0, \pi) \backslash J) \leq \varepsilon$.

(ii) If $\theta \in J$, then for any family $\mathcal{Q}^{\prime} \subset \mathcal{Q}$ there exists a subcollection $\mathcal{Q}_{1}$ of $\mathcal{Q}^{\prime}$ of cardinality at least

$$
\delta \varepsilon\left(\frac{\left|\mathcal{Q}^{\prime}\right|}{|\mathcal{Q}|}\right)^{2}|\mathcal{Q}|
$$

such that the elements of the family $\left\{\operatorname{proj}_{\theta}(Q): Q \in \mathcal{Q}_{1}\right\}$ are $\rho$ separated.

(iii) $J$ is a finite union of open intervals.

Proof. Throughout the course of the proof, $C_{i}$ denotes a constant which depends only on $A, A_{1}, A_{2}$ and $\gamma$. Let $E$ be the union of the elements in $\mathcal{Q}$, and let $\mu$ be normalized Lebesgue measure on $E$. Consider the Riesz 1-energy of $\mu$,

$$
I_{1}(\mu)=\iint|x-y|^{-1} d \mu(x) d \mu(y) .
$$


From [14, Proposition 7] we have

$$
I_{1}(\mu) \leq C_{1} \rho^{\gamma-1} .
$$

Combining this with [9, Theorem 9.7] yields

$$
\int_{0}^{\pi}\left\|d \mu_{\theta} / d x\right\|_{2}^{2} d \theta \leq C_{2} I_{1}(\mu) \leq C_{3} \rho^{\gamma-1},
$$

where $\mu_{\theta}$ is the push forward of $\mu$ under the map $P_{\theta}=R_{-\theta} \circ \operatorname{proj}_{\theta}$, where $R_{-\theta}$ denotes rotation of angle $-\theta$ about the origin. Here $\|\cdot\|_{2}$ denotes the usual $L^{2}$ norm.

Let $f_{\theta}=d \mu_{\theta} / d x$ denote the density of $\mu_{\theta}$. We claim that $\theta \mapsto\left\|f_{\theta}\right\|_{2}$ is continuous. By the dominated convergence theorem, it suffices to show that the density $f_{\theta}(x)$ is continuous for all $x$. Since

$$
f_{\theta}(x)=\sum_{Q \in \mathcal{Q}} f_{\theta, Q}(x),
$$

where $f_{\theta, Q}$ is the density of the push forward of the measure $\left.\mu\right|_{Q}$ under $P_{\theta}$, it is enough to verify that each $f_{\theta, Q}$ is continuous, which follows from the convexity of $Q$. Let

$$
J=\left\{\theta:\left\|f_{\theta}\right\|_{2}^{2}<\varepsilon^{-1} C_{3} \rho^{\gamma-1}\right\} .
$$

From (2.1) we deduce that $\mathcal{L}([0, \pi) \backslash J) \leq \varepsilon$. Since $\left\|f_{\theta}\right\|_{2}$ depends continuously on $\theta, J$ is open and therefore a finite union of open intervals. Hence it remains to prove (ii).

Fix a non-empty subfamily $\mathcal{Q}^{\prime}$ of $\mathcal{Q}$ and write $\eta=\left|\mathcal{Q}^{\prime}\right| /|\mathcal{Q}|$. Let $\nu$ be the restriction of $\mu$ to the union of the elements of $\mathcal{Q}^{\prime}$, and let $\left\{\nu_{\theta}\right\}$ be the corresponding projections of $\nu$, and $\left\{f_{\theta}^{\prime}\right\}$ their densities. As each element $Q \in \mathcal{Q}$ contains a disk of radius $A^{-1} \rho$, and is contained in a disk of radius $A \rho$, we have $\mu(Q) / \mu\left(Q^{\prime}\right) \geq A^{-4}$ for all $Q, Q^{\prime} \in \mathcal{Q}$. Hence, $\nu$ has total mass at least $A^{-4} \eta$. It is also clear that

$$
\left\|f_{\theta}^{\prime}\right\|_{2}^{2} \leq\left\|f_{\theta}\right\|_{2}^{2}<\varepsilon^{-1} C_{3} \rho^{\gamma-1}
$$

for all $\theta \in J$. On the other hand, using the Cauchy-Schwarz inequality,

$$
A^{-4} \eta \leq \nu\left(\mathbb{R}^{2}\right)=\nu_{\theta}\left(\operatorname{Supp}\left(\nu_{\theta}\right)\right) \leq \mathcal{L}\left(\operatorname{Supp}\left(\nu_{\theta}\right)\right)^{1 / 2}\left\|f_{\theta}^{\prime}\right\|_{2} .
$$

From (2.2) and (2.3) we deduce that if $\theta \in J$ then

$$
\mathcal{L}\left(\operatorname{Supp}\left(\nu_{\theta}\right)\right) \geq C_{4} \varepsilon \eta^{2} \rho^{1-\gamma} .
$$

For such $\theta \in J$ we may select a set of points $E_{\theta} \subset \operatorname{Supp}\left(\nu_{\theta}\right)$ of cardinality at least $\delta \varepsilon \eta^{2}|\mathcal{Q}|$, for which the elements are $(2 A+1) \rho$-separated, where $\delta=C_{4} /(2(2 A+1))$. For each $x \in E_{\theta}$ choose an element $Q_{x} \in \mathcal{Q}^{\prime}$ for which $x \in P_{\theta}\left(Q_{x}\right)$. Since $P_{\theta}\left(Q_{x}\right)$ is contained in the interval $[x-A \rho, x+A \rho]$, we see that that the family $\left\{P_{\theta}\left(Q_{x}\right): x \in E_{\theta}\right\}$ is $\rho$-separated. This concludes the proof, as $\left\{Q_{x}\right\}_{x \in E_{\theta}}$ has the desired properties. 
3. Proof of Proposition 1.2. Throughout this section, $\Lambda$ is the attractor of a homogeneous uniform-fibre Gatzouras-Lalley construction:

$$
\Lambda=\bigcup_{(i, j) \in D} S_{i j}(\Lambda)
$$

where $D=\{1, \ldots, m\} \times\{1, \ldots, n\}$ and $S_{i j}(x, y)=\left(f_{i j}(x), g_{i}(y)\right)$ with $f_{i j}(x)=a x+c_{i j}$ and $g_{i}(x)=b x+d_{i}$ for some $0<a<b<1, c_{i j}, d_{i} \in \mathbb{R}$.

We fix some notation. For a positive integer $N$, let

$$
\Sigma_{N}=\left\{\sigma=\sigma_{1} \sigma_{2} \cdots: 0 \leq \sigma_{k}<N \text { for all } k \in \mathbb{N}\right\},
$$

i.e. the set of one-sided infinite strings over the alphabet $\{0,1, \ldots, N-1\}$, and let $\Sigma_{N}^{*}$ be the collection of all finite strings. We will also let

$$
\Sigma_{N}^{k}=\left\{\sigma=\sigma_{1} \cdots \sigma_{k}: 0 \leq \sigma_{j}<N \text { for all } 1 \leq j \leq k\right\} .
$$

For a string $\sigma=\sigma_{1} \sigma_{2} \cdots \in \Sigma_{N}$ and a natural number $k \in \mathbb{N}$, let $\left.\sigma\right|_{k}=$ $\sigma_{1} \cdots \sigma_{k}$ denote the truncation of $\sigma$ to its first $k$ symbols. For a string $\sigma=$ $\sigma_{1} \cdots \sigma_{k} \in \Sigma_{N}^{*}$ of finite length, we let $|\sigma|=k$ denote the length of the string. For $\sigma, \eta \in \Sigma_{N}^{*}$ we denote their concatenation by $\sigma \eta=\sigma_{1} \cdots \sigma_{|\sigma|} \eta_{1} \cdots \eta_{|\eta|}$.

This notation gives rise to a natural coding of the set $\Lambda$ : Let

$$
X_{k}=\left\{\left(\eta, \eta^{\prime}\right) \in \Sigma_{m}^{k} \times \Sigma_{n}^{k}:\left(\eta_{i}, \eta_{i}^{\prime}\right) \in D \text { for } i=1, \ldots, k\right\},
$$

and write

$$
f_{\sigma, \sigma^{\prime}}=f_{\sigma_{1}, \sigma_{1}^{\prime}} \cdots f_{\sigma_{k}, \sigma_{k}^{\prime}}, \quad g_{\xi}=g_{\xi_{1}} \cdots g_{\xi_{|\xi|}}
$$

for $\left(\sigma, \sigma^{\prime}\right) \in X_{k}$ and $\xi \in \Sigma_{m}^{*}$. For $\sigma \in \Sigma_{m}^{*}$ and $\left(\xi, \sigma^{\prime}\right) \in X_{k}$ we set

$$
Q\left(\sigma, \xi, \sigma^{\prime}\right)=f_{\xi, \sigma^{\prime}}[0,1] \times g_{\sigma}[0,1] .
$$

Then we may write

$$
\Lambda=\bigcap_{k \geq 1} \bigcup_{\left(\sigma, \sigma^{\prime}\right) \in X_{k}} Q\left(\sigma, \sigma, \sigma^{\prime}\right) .
$$

It is easy to see that, in the homogeneous uniform fibre case,

$$
\gamma:=\operatorname{dim}_{\mathrm{H}}(\Lambda)=\frac{\log m}{-\log b}+\frac{\log n}{-\log a} .
$$

If $\gamma \geq 1$, then for any $\varepsilon>0$ we can find a subset $\Lambda_{\varepsilon} \subset \Lambda$, which is also the invariant set of an irrational, homogeneous uniform fibre construction, and such that $1-\varepsilon<\operatorname{dim}_{H}\left(\Lambda_{\varepsilon}\right)<1$. Indeed, all we need to do is iterate the original iterated function system a large number of times, remove an appropriate subset of maps from the resulting system, and let $\Lambda_{\varepsilon}$ be the attractor of the reduced system. (It follows from (3.1) that by this procedure we can obtain sets of dimension arbitrarily close to any prefixed value not exceeding $\gamma$.) Thus, by replacing $\Lambda$ with $\Lambda_{\varepsilon}$ and then letting $\varepsilon \rightarrow 0$, we can assume without loss of generality that $\gamma<1$, and we will do so for the rest of the proof. 
For a positive integer $k$, set $\ell(k)=\max \left\{k^{\prime}: b^{k} \leq a^{k^{\prime}}\right\}$ and $Z_{k}=$ $a^{\ell(k)} b^{-k} \in\left[1, a^{-1}\right)$. Given $\xi \in \Sigma_{m}^{\ell(k)}$ and $\xi^{\prime} \in \Sigma_{m}^{\ell(k)+1}$ we set

$$
\begin{aligned}
\mathcal{Q}_{k}(\xi) & =\left\{Q\left(\sigma, \xi, \sigma^{\prime}\right):\left(\sigma, \sigma^{\prime}\right) \in \Sigma_{m}^{k} \times \Sigma_{n}^{\ell(k)}\right\}, \\
\widetilde{\mathcal{Q}}_{k}\left(\xi^{\prime}\right) & =\left\{Q\left(\sigma, \xi^{\prime}, \sigma^{\prime}\right):\left(\sigma, \sigma^{\prime}\right) \in \Sigma_{m}^{k} \times \Sigma_{n}^{\ell(k)+1}\right\} .
\end{aligned}
$$

We note that $\left(\xi, \sigma^{\prime}\right) \in X_{\ell(k)}$ and therefore $\mathcal{Q}_{k}(\xi)$ is well defined, and likewise $\widetilde{\mathcal{Q}}_{k}\left(\xi^{\prime}\right)$. Due to our choice of $\ell(k)$, and in particular the fact that $Z_{k}$ is bounded, the elements of $\mathcal{Q}_{k}(\xi)$ and $\widetilde{\mathcal{Q}}_{k}\left(\xi^{\prime}\right)$ are approximately squares, which project onto intervals of size $\approx b^{k}$, i.e. there exists a constant $c>1$ such that $c^{-1} \leq \operatorname{diam}\left(\operatorname{proj}_{\theta}(Q)\right) / b^{k} \leq c$ for all $Q \in \mathcal{Q}_{k}(\xi)$.

For each $\xi \in \Sigma_{m}^{\ell(k)}$ we may apply Theorem 2.1 to the family $\mathcal{Q}_{k}(\xi)$ to obtain a "large" set of "good" angles $J_{\xi}$. We remark that in the context of [14, Theorem 1], the sets $\mathcal{Q}_{k}(\xi)$ are all translates of each other thanks to the product structure. In particular, all $J_{\xi}$ are equal and hence there is a "large" set of "good" angles which is uniform in $\xi$. However, in our situation we have little information about the intersection of the sets $J_{\xi}$, or even the cardinality of the set $\left\{\xi \in \Sigma_{m}^{\ell(k)}: \theta \in J_{\xi}\right\}$ for a fixed $\theta \in[0, \pi)$. The reduction to the uniform horizontal fibre case and the modification of the discrete Marstrand Theorem given in Theorem 2.1 are needed in order to tackle this additional difficulty. Another step in this direction is the following lemma, which shows that there exists a "large" set of angles $J \subset[0, \pi)$ such that whenever $\theta \in J$, then $\theta \in J_{\xi}$ for a "large" number of $\xi \in \Sigma_{m}^{\ell(k)}$.

LEMma 3.1. There exists a constant $\delta>0$ such that for any $k \in \mathbb{N}$ and $\varepsilon>0$, there exists a set $J \subset[0, \pi)$ with the following properties:

(i) $\mathcal{L}([0, \pi) \backslash J) \leq \varepsilon^{1 / 2}$.

(ii) If $\theta \in J$ then there exists a subcollection $\mathcal{B} \subset \Sigma_{m}^{\ell(k)}$ with the following properties:

(a) The cardinality of $\mathcal{B}$ is at least $\left(1-\varepsilon^{1 / 2}\right) m^{\ell(k)}$.

(b) For any $\xi \in \mathcal{B}$ and any family $\mathcal{Q}^{\prime} \subset \mathcal{Q}_{k}(\xi)$, there exists a subcollection $\mathcal{Q}_{1}$ of $\mathcal{Q}^{\prime}$ of cardinality at least

$$
\delta \varepsilon\left(\frac{\left|\mathcal{Q}^{\prime}\right|}{\left|\mathcal{Q}_{k}(\xi)\right|}\right)^{2} m^{k \gamma}
$$

for which the elements of the family $\left\{\operatorname{proj}_{\theta}(Q): Q \in \mathcal{Q}_{1}\right\}$ are $b^{k}$-separated.

(iii) $J$ is a finite union of open intervals.

Proof. We first show that for a fixed $\xi \in \Sigma_{m}^{\ell(k)}$ the family $\mathcal{Q}_{k}(\xi)$ satisfies the hypothesis of Theorem 2.1. Each $Q \in \mathcal{Q}_{k}(\xi)$ has size $Z_{k} b^{k} \times b^{k}$, thus taking $A=\sqrt{1+a^{-2}}$ implies that $Q$ is contained inside some ball of radius $A b^{k}$ 
with centre in $Q$. Similarly, $Q$ contains a ball of radius $A^{-1} b^{k}$. In addition,

$$
\left|\mathcal{Q}_{k}(\xi)\right|=m^{k} n^{\ell(k)}>n^{-1}\left(n^{\log _{a} b} m\right)^{k}=n^{-1} b^{-k \gamma}=A_{1}^{-1} b^{-k \gamma} .
$$

Finally fix $j<k$, and let $Q=Q\left(\sigma,\left.\xi\right|_{\ell(j)}, \sigma^{\prime}\right) \in \mathcal{Q}_{j}\left(\left.\xi\right|_{\ell(j)}\right)$. Then

$$
\begin{aligned}
& \left|\left\{Q\left(\eta, \xi, \eta^{\prime}\right) \in \mathcal{Q}_{k}(\xi): Q\left(\eta, \xi, \eta^{\prime}\right) \subset Q\right\}\right| \\
& =\left|\left\{\left(\eta, \eta^{\prime}\right) \in \Sigma_{m}^{k} \times \Sigma_{n}^{\ell(k)}:\left.\eta\right|_{\ell(j)}=\sigma,\left.\eta^{\prime}\right|_{j}=\sigma^{\prime}\right\}\right| \\
& =m^{k-j} n^{\ell(k)-\ell(j)}<n b^{-(k-j) \gamma} .
\end{aligned}
$$

Any ball $B$ of radius $b^{j}$ may intersect at most nine elements of $\mathcal{Q}_{j}\left(\left.\xi\right|_{\ell(j)}\right)$. Combining this with (3.3) and setting $A_{2}=9 n$, we see that $B$ may intersect at most $A_{2} b^{-(k-j) \gamma}$ elements of $\mathcal{Q}_{k}(\xi)$. It is worth noting that the constants $A, A_{1}, A_{2}, \gamma$ have no dependence on the choice of $\xi$.

The above argument combined with Theorem 2.1 implies the following: there is $\delta>0$ such that for any $\varepsilon>0, k \in \mathbb{N}$ and $\xi \in \Sigma_{m}^{\ell(k)}$, there exists a set of angles $J_{\xi} \subset[0, \pi)$ satisfying:

(i) $\mathcal{L}\left([0, \pi) \backslash J_{\xi}\right) \leq \varepsilon$.

(ii) If $\theta \in J_{\xi}$, then for any family $\mathcal{Q}^{\prime} \subset \mathcal{Q}_{k}(\xi)$, there exists a subcollection $\mathcal{Q}_{1}$ of $\mathcal{Q}^{\prime}$ of cardinality at least

$$
\delta \varepsilon\left(\frac{\left|\mathcal{Q}^{\prime}\right|}{\left|\mathcal{Q}_{k}(\xi)\right|}\right)^{2}\left|\mathcal{Q}_{k}(\xi)\right|
$$

such that the elements of the family $\left.\operatorname{sroj}_{\theta}(Q): Q \in \mathcal{Q}_{1}\right\}$ are $b^{k}$ separated.

(iii) $J_{\xi}$ is a finite union of open intervals.

Furthermore combining (3.2) with property (ii) above, we may take the cardinality of $\mathcal{Q}_{1}$ to be at least $A_{1}^{-1} \delta \varepsilon\left(\left|\mathcal{Q}^{\prime}\right| /\left|\mathcal{Q}_{k}(\xi)\right|\right)^{2} b^{-k \gamma}$.

For $\theta \in[0, \pi)$, let $\Xi_{\theta}=\left\{\xi \in \Sigma_{m}^{\ell(k)}: \theta \in J_{\xi}\right\}$. Then

$$
\int_{0}^{\pi}\left|\Sigma_{m}^{\ell(k)} \backslash \Xi_{\theta}\right| d \theta=\sum_{\xi \in \Sigma_{m}^{\ell(k)}} \mathcal{L}\left([0, \pi) \backslash J_{\xi}\right)<m^{\ell(k)} \varepsilon .
$$

We set

$$
J=\left\{\theta \in[0, \pi):\left|\Xi_{\theta}\right|>\left(1-\varepsilon^{1 / 2}\right) m^{\ell(k)}\right\} .
$$

Then from (3.4) we deduce $\mathcal{L}([0, \pi) \backslash J) \leq \varepsilon^{1 / 2}$. Finally, we may express $J$ in the form

$$
J=\bigcup_{|B|>\left(1-\varepsilon^{1 / 2}\right) m^{\ell(k)}} \bigcap_{\xi \in B} J_{\xi},
$$

which is easily seen to be a finite union of open intervals. This completes the proof. 
Given $\tau \in \mathbb{R}$, let $\Pi_{\tau}(x, y)=a^{-\tau} x+y$ and $\widetilde{\Pi}_{\tau}(x, y)=-a^{-\tau} x+y$. Note that for a fixed $\theta \in(0, \pi / 2)$, the set $\operatorname{proj}_{\theta}(\Lambda)$ is affinely equivalent to $\Pi_{\tau}(\Lambda)$, where $\tau=\log (\tan (\theta)) / \log a$. Similarly, for $\theta \in(\pi / 2, \pi)$ the set $\operatorname{proj}_{\theta}(\Lambda)$ is affinely equivalent to $\widetilde{\Pi}_{\tau}(\Lambda)$ for $\tau=\log (-\tan (\theta)) / \log a$. For technical reasons, it is convenient to work with the maps $\Pi_{\tau}$ in place of the orthogonal projections $\operatorname{proj}_{\theta}$. Thus, as affine bijections preserve Hausdorff dimension, it suffices to show that

$$
\operatorname{dim}_{\mathrm{H}}\left(\Pi_{\tau}(\Lambda)\right), \operatorname{dim}_{\mathrm{H}}\left(\widetilde{\Pi}_{\tau}(\Lambda)\right) \geq \gamma \quad \text { for any } \tau \in \mathbb{R} .
$$

The proofs for $\Pi_{\tau}$ and $\widetilde{\Pi}_{\tau}$ are analogous, and accordingly we will present the proof only for $\Pi_{\tau}$, corresponding to $\theta \in(0, \pi / 2)$.

Since the map $\theta \mapsto \log (\tan (\theta)) / \log a$ is a $C^{1}$ injection on any compact subset of $(0, \pi / 2)$, a reparametrization of Lemma 3.1 yields the following:

COROllary 3.2. Given $\tau \in \mathbb{R}$, there exist constants $\delta^{\prime}, L>0$ such that for any $k \in \mathbb{N}$ and $\varepsilon>0$ there is a set $\widetilde{F} \subset[\tau, \tau+1)$ with the following properties:

(i) $\mathcal{L}([\tau, \tau+1) \backslash \widetilde{F}) \leq L \varepsilon^{1 / 2}$.

(ii) If $t \in \widetilde{F}$, then there exists a subcollection $\mathcal{B}_{t} \subset \Sigma_{m}^{\ell(k)}$ of cardinality at least $\left(1-\varepsilon^{1 / 2}\right) m^{\ell(k)}$ such that for any $\xi \in \mathcal{B}_{t}$ and any family $\mathcal{Q}^{\prime} \subset \mathcal{Q}_{k}(\xi)$, there exists a subcollection $\mathcal{Q}_{1}$ of $\mathcal{Q}^{\prime}$ of cardinality at least

$$
\delta^{\prime} \varepsilon\left(\frac{\left|\mathcal{Q}^{\prime}\right|}{\left|\mathcal{Q}_{k}(\xi)\right|}\right)^{2} b^{-k \gamma}
$$

for which the elements of the family $\left\{\Pi_{t}(Q): Q \in \mathcal{Q}_{1}\right\}$ are $b^{k}-$ separated.

(iii) $\widetilde{F}$ is a finite union of open intervals.

Property (ii) of Corollary 3.2 holds for the families $\widetilde{\mathcal{Q}}_{k}\left(\xi^{\prime}\right)$, as each element is contained in an element of $\mathcal{Q}_{k}(\xi)$ with $\left.\xi^{\prime}\right|_{\ell(k)}=\xi$. For $t \in[\tau, \tau+1)$, let

$$
\widetilde{\mathcal{B}}_{t}=\left\{\xi \xi^{\prime}: \xi \in \mathcal{B}_{t}, \xi^{\prime} \in \Sigma_{m}\right\} \subset \Sigma_{m}^{\ell(k)+1}
$$

be the collection of strings obtained by adjoining an element of $\Sigma_{m}$ to those of $\mathcal{B}_{t}$.

We now construct a branching process driven by an irrational rotation, which we will then use to construct a subset $\Lambda_{\tau}$ of $\Pi_{\tau}(\Lambda)$ satisfying $\operatorname{dim}_{\mathrm{H}}\left(\Lambda_{\tau}\right) \geq \gamma$. Define a map $T:[0,1) \rightarrow[0,1)$ by $T(x)=x+\alpha \bmod 1$, where $\alpha=\log Z_{k} /(-\log a)=k \log _{a} b-\ell(k) \in[0,1)$. We may choose a sequence $\left\{e_{j}\right\}_{j}$ to satisfy

$$
T^{j}(0)=\frac{\log \left(a^{e_{j}} b^{-j k}\right)}{-\log a}
$$


i.e. set $e_{1}=\ell(k)$, and for $j>1$ let

$$
e_{j}= \begin{cases}e_{j-1}+\ell(k) & \text { if } a^{e_{j-1}+\ell(k)+1}<b^{j k} \\ e_{j-1}+\ell(k)+1 & \text { otherwise. }\end{cases}
$$

For $j \in \mathbb{N}$ let

$$
\Gamma_{k}(j)= \begin{cases}\mathcal{B}_{t} & \text { if } t=\tau+T^{j}(0) \in \widetilde{F} \text { and } e_{j+1}-e_{j}=\ell(k), \\ \widetilde{\mathcal{B}_{t}} & \text { if } t=\tau+T^{j}(0) \in \widetilde{F} \text { and } e_{j+1}-e_{j}=\ell(k)+1, \\ \Sigma_{m}^{e_{j+1}-e_{j}} & \text { otherwise. }\end{cases}
$$

Then, by Corollary 3.2 .

$$
\left|\Gamma_{k}(j)\right|>\left(1-\varepsilon^{1 / 2}\right) m^{e_{j+1}-e_{j}} .
$$

We now introduce a function $s: \mathbb{N} \rightarrow \mathbb{N}$ which relates the two quantities $e_{j}$ and $j k$. For a positive integer $j$, let $s(j)$ be the unique integer such that $e_{s(j)-1}<j k \leq e_{s(j)}$. Then from 3.5 we see

$$
j \frac{\log a}{\log b} \leq s(j)<j \frac{\log a}{\log b}+\frac{\log a}{k \log b}+1 .
$$

For $j \in \mathbb{N}$ we let

$$
\begin{aligned}
& \Delta_{k}(j)=\left\{\eta \in \Sigma_{m}^{j k-e_{s(j)-1}}: \eta \eta^{\prime} \in \Gamma_{k}(s(j)-1) \text { for some } \eta^{\prime} \in \Sigma_{m}^{e_{s(j)}-j k}\right\}, \\
& \Delta_{k}^{\prime}(j)=\left\{\eta^{\prime} \in \Sigma_{m}^{e_{s(j)}-j k}: \eta \eta^{\prime} \in \Gamma_{k}(s(j)-1) \text { for some } \eta \in \Sigma_{m}^{j k-e_{s(j)-1}}\right\} .
\end{aligned}
$$

In the event that $j k=e_{s(j)}$, we take $\Delta_{k}^{\prime}(j)$ to contain the empty word, otherwise, both $\Delta_{k}(j)$ and $\Delta_{k}^{\prime}(j)$ are non-empty and, moreover, from (3.6) we deduce that

$$
\left|\Delta_{k}(j)\right|>\left(1-\varepsilon^{1 / 2}\right) m^{j k-e_{s(j)-1}}, \quad\left|\Delta_{k}^{\prime}(j)\right|>\left(1-\varepsilon^{1 / 2}\right) m^{e_{s(j)}-j k} .
$$

For $\eta \in \Sigma_{m}^{j k-e_{s(j)-1}}$, set

$$
\phi_{j}(\eta)=\left|\left\{\eta^{\prime} \in \Delta_{k}^{\prime}(j): \eta \eta^{\prime} \in \Gamma_{k}(s(j)-1)\right\}\right| .
$$

Then by letting

$$
\Theta(j):=\left\{\eta \in \Delta_{k}(j): \phi_{j}(\eta)>\frac{1}{2} m^{e_{s}(j)-j k}\right\},
$$

we deduce that

$$
\begin{aligned}
& \left|\Sigma_{m}^{e_{s(j)}-e_{s(j)-1}} \backslash \Gamma_{k}(s(j)-1)\right|=\sum_{\eta \in \Sigma_{m}^{j k-e_{s(j)-1}}}\left(m^{e_{s(j)}-j k}-\phi_{j}(\eta)\right) \\
& >\sum_{\eta \in \Sigma_{m}^{j k-e_{s(j)-1} \backslash \Theta(j)}}\left(m^{e_{s(j)}-j k}-\phi_{j}(\eta)\right)>\frac{1}{2} m^{e_{s(j)}-j k}\left|\Sigma_{m}^{j k-e_{s(j)-1}} \backslash \Theta(j)\right| .
\end{aligned}
$$


Combining this with 3.6 we see that $|\Theta(j)|>\left(1-2 \varepsilon^{1 / 2}\right) m^{j k-e_{s(j)-1}}$. For $\eta \in \Theta(j)$, let

$$
\Theta^{\prime}(j, \eta)=\left\{\eta^{\prime}: \eta \eta^{\prime} \in \Gamma_{k}(s(j)-1)\right\}
$$

and

$$
\begin{aligned}
G_{k}(j, \eta)=\left\{\eta^{\prime} \xi_{1} \cdots \xi_{s(j+1)-s(j)-1} \eta^{\prime \prime}:\right. & \eta^{\prime} \in \Theta^{\prime}(j, \eta), \\
\eta^{\prime \prime} & \left.\in \Theta(j+1), \xi_{i} \in \Gamma_{k}(i+s(j)-1)\right\},
\end{aligned}
$$

Then from (3.6), (3.7) and the definitions of $\Delta_{k}(j)$ and $\Delta_{k}^{\prime}(j)$, we see that if $\varepsilon<1 / 9$, then

$$
\begin{aligned}
\left|G_{k}(j, \eta)\right| & =|\Theta(j+1)|\left|\Theta^{\prime}(j, \eta)\right| \prod_{i=s(j)}^{s(j+1)-2}\left|\Gamma_{k}(i)\right| \\
& >\frac{1}{2}\left(1-2 \varepsilon^{1 / 2}\right)\left(1-\varepsilon^{1 / 2}\right)^{s(j+1)-s(j)-1} m^{k} \\
& >\frac{1}{6}\left(1-\varepsilon^{1 / 2}\right)^{s(j+1)-s(j)-1} m^{k}>\frac{1}{6}\left(\frac{2}{3}\right)^{2 \frac{\log a}{\log b} m^{k} .}
\end{aligned}
$$

For $\xi \in \Sigma_{m}^{e_{j+1}-e_{j}}$ and $\eta \in \Theta(j)$ let

$$
\begin{aligned}
M_{k}(j, \eta) & =G_{k}(j, \eta) \times \Sigma_{n}^{e_{j+1}-e_{j}} \\
\mathcal{U}_{k}(j, \xi, \eta) & =\left\{Q\left(\sigma, \xi, \sigma^{\prime}\right):\left(\sigma, \sigma^{\prime}\right) \in M_{k}(j, \eta)\right\} .
\end{aligned}
$$

Then for any $\xi \in \Sigma_{m}^{e_{j+1}-e_{j}}$ and $\eta \in \Theta(j)$ such that $e_{j+1}-e_{j}=\ell(k), 3.8$ yields

$$
\left|\mathcal{U}_{k}(j, \xi, \eta)\right|=n^{\ell(k)}\left|G_{k}(j, \eta)\right|>\frac{1}{6}\left(\frac{2}{3}\right)^{2 \frac{\log a}{\log b}} n^{\ell(k)} m^{k}>c\left|\mathcal{Q}_{k}(\xi)\right|
$$

for some constant $c$, independent of $k$ and $\varepsilon$. Similarly, if $e_{j+1}-e_{j}=\ell(k)+1$ we have

$$
\left|\mathcal{U}_{k}(j, \xi, \eta)\right|>c\left|\widetilde{\mathcal{Q}}_{k}(\xi)\right|
$$

Thus if $t=T^{j}(0)+\tau \in \widetilde{F}, \xi \in \Gamma_{k}(j)$ and $\eta \in \Theta(j)$, by Corollary 3.2 there exists a subcollection $\mathcal{U}_{k}^{\prime}(j, \xi, \eta)$ of $\mathcal{U}_{k}(j, \xi, \eta)$, of cardinality at least $c^{2} \delta^{\prime} \varepsilon b^{-k \gamma}$, for which the elements of the family $\left\{\Pi_{t}(Q): Q \in \mathcal{U}_{k}^{\prime}(j, \xi, \eta)\right\}$ are $b^{k}$-separated. We let $M_{k}^{\prime}(j, \xi, \eta)$ denote the subset of $M_{k}(j, \eta)$ for which

$$
\mathcal{U}_{k}^{\prime}(j, \xi, \eta)=\left\{Q\left(\sigma, \xi, \sigma^{\prime}\right):\left(\sigma, \sigma^{\prime}\right) \in M_{k}^{\prime}(j, \xi, \eta)\right\} .
$$

Lemma 3.3. There exists a rooted tree, $\mathcal{R}$, with vertices labelled by elements of $\Sigma_{m}^{j k} \times \Sigma_{n}^{e_{j}}$ for some $j \in \mathbb{N}$, with the following properties: Denote the elements of the $j$ th level by $\mathcal{R}_{j}$. Then:

A: If $\left(\sigma, \sigma^{\prime}\right)$ is the parent of $\left(\eta, \eta^{\prime}\right)$ then $\left(\left.\eta\right|_{|\sigma|},\left.\eta^{\prime}\right|_{\left|\sigma^{\prime}\right|}\right)=\left(\sigma, \sigma^{\prime}\right)$.

B: If $\left(\sigma, \sigma^{\prime}\right) \in \mathcal{R}_{j}$ then $Q\left(\sigma,\left.\sigma\right|_{\left|\sigma^{\prime}\right|}, \sigma^{\prime}\right)$ has size $a^{-T^{j}(0)} b^{j k} \times b^{j k}$. 
C: If $\left(\sigma, \sigma^{\prime}\right) \in \mathcal{R}_{j}$ then

$$
\begin{aligned}
& \xi_{i}(\sigma):=\sigma_{e_{i}+1} \sigma_{e_{i}+2} \cdots \sigma_{e_{i+1}} \in \Gamma_{k}(i) \quad \text { for } j \leq i \leq s(j)-2, \\
& \eta\left(\sigma^{\prime}\right):=\sigma_{e_{s(j)-1}+1} \sigma_{e_{s(j)-1}+2} \cdots \sigma_{j k} \in \Theta(j) .
\end{aligned}
$$

D: The elements of the set $\left\{\Pi_{\tau} Q\left(\sigma,\left.\sigma\right|_{\left|\sigma^{\prime}\right|}, \sigma^{\prime}\right):\left(\sigma, \sigma^{\prime}\right) \in \mathcal{R}_{j}\right\}$ are disjoint and $b^{j k}$-separated.

E: Each element of $\mathcal{R}_{j}$ has the same number of offspring $C_{j}$; moreover

$$
\begin{aligned}
& T^{j}(0)+\tau \in \widetilde{F} \Rightarrow C_{j}>c^{2} \delta^{\prime} \varepsilon b^{-k \gamma}, \\
& T^{j}(0)+\tau \notin \widetilde{F} \Rightarrow C_{j}=1 .
\end{aligned}
$$

Proof. At each stage of the construction we define the offspring $C\left(\sigma, \sigma^{\prime}\right)$ of the element $\left(\sigma, \sigma^{\prime}\right) \in \mathcal{R}_{j}$ to be the concatenation of $\left(\sigma, \sigma^{\prime}\right)$ with elements of $\mathcal{U}_{k}(\xi, j)$, where $\xi=\xi_{j}(\sigma)$. Accordingly, we require $\left(\sigma, \sigma^{\prime}\right) \in \mathcal{R}_{j}$ to be such that $|\sigma|-\left|\sigma^{\prime}\right|>\ell(k)$. Therefore we will start the induction at $j_{0} \in \mathbb{N}$, where $\ell(k)<j_{0} k-e_{j_{0}}$.

To construct $\mathcal{R}_{j_{0}}$, let $\left(\sigma, \sigma^{\prime}\right)$ be any element of $\Sigma_{m}^{e_{j_{0}}} \times \Sigma_{n}^{e_{j_{0}}}$, and for $i=j_{0}, j_{0}+1, \ldots, s\left(j_{0}\right)-2$ let $\xi_{i} \in \Gamma_{k}(i)$. Then set

$$
\mathcal{R}_{j_{0}}=\left\{\left(\sigma \xi_{j_{0}} \xi_{j_{0}+1} \cdots \xi_{s\left(j_{0}\right)-2} \eta, \sigma^{\prime}\right)\right\},
$$

where $\eta$ is any element of $\Theta\left(j_{0}\right)$. Properties $\mathbf{A}, \mathbf{D}$ and $\mathbf{E}$ follow trivially. To see $\mathbf{B}$, note that the rectangle $Q\left(\sigma \xi_{j_{0}} \xi_{j_{0}+1} \cdots \xi_{s\left(j_{0}\right)-2} \eta, \sigma, \sigma^{\prime}\right)$ has size $a^{e_{j_{0}}} \times b^{j_{0} k}=a^{-T^{j_{0}}(0)} b^{j_{0} k} \times b^{j_{0} k}$. By construction $\xi_{i}\left(\sigma \xi_{j_{0}} \xi_{j_{0}+1} \cdots \xi_{s\left(j_{0}\right)-2} \eta\right)=$ $\xi_{i} \in \Gamma_{k}(i)$, for $i=j_{0}, j_{0}+1, \ldots, s\left(j_{0}\right)-2$, and $\eta\left(\sigma \xi_{j_{0}} \xi_{j_{0}+1} \cdots \xi_{s\left(j_{0}\right)-2} \eta\right)=$ $\eta \in \Theta(j)$, showing $\mathbf{C}$.

Now suppose that for $j>j_{0}, \mathcal{R}_{j}$ has been defined and properties $\mathbf{A}-\mathbf{E}$ hold. We now construct $\mathcal{R}_{j+1}$. Fix an element $\left(\sigma, \sigma^{\prime}\right) \in \mathcal{R}_{j}$, and let $\xi=\xi_{j}(\sigma)$, $\eta=\eta(\sigma)$. In the case that $T^{j}(0)+\tau \in \widetilde{F}$ let the offspring $C\left(\sigma, \sigma^{\prime}\right)$ of $\left(\sigma, \sigma^{\prime}\right)$ be

$$
C\left(\sigma, \sigma^{\prime}\right)=\left\{\left(\sigma \nu, \sigma^{\prime} \nu^{\prime}\right):\left(\nu, \nu^{\prime}\right) \in M_{k}^{\prime}(j, \xi, \eta)\right\},
$$

otherwise $T^{j}(0)+\tau \notin \widetilde{F}$, in which case we let $C(\sigma, \sigma)=\left\{\left(\sigma \nu, \sigma^{\prime} \nu^{\prime}\right)\right\}$ where $\left(\nu, \nu^{\prime}\right)$ is any element of $M_{k}(j, \eta)$. Property $\mathbf{A}$ is clear. Any element of $\mathcal{R}_{j+1}$ has size

$$
a^{e_{j+1}} \times b^{(j+1) k}=a^{-T^{j+1}(0)} b^{(j+1) k} \times b^{(j+1) k},
$$

which shows B. If $\left(\sigma \nu, \sigma^{\prime} \nu^{\prime}\right) \in C\left(\sigma, \sigma^{\prime}\right)$ then as $\mathcal{R}_{j}$ satisfies property $\mathbf{C}$, we have $\xi_{i}(\sigma \nu)=\xi_{i}(\sigma) \in \Gamma_{j}(i)$ for $i=j+1, j+2, \ldots, s(j)-2$ and $\eta=\eta(\sigma) \in$ $\Theta(j)$. Further, $\left(\nu, \nu^{\prime}\right) \in M_{k}^{\prime}(j, \xi, \eta)$ and so $\xi_{i}(\sigma \nu) \in \Gamma_{k}(i)$ for $i=s(j), s(j)+$ $1, \ldots, s(j+1)-2$, and $\eta(\sigma \nu) \in \Theta(j+1)$. Finally, $\xi_{s(j)-1}(\sigma \nu)=\eta \eta^{\prime}$, where $\eta \in \Theta(j)$ and $\eta^{\prime}=\nu_{1} \nu_{2} \cdots \nu_{s(j)-j k} \in \Theta^{\prime}(j+1, \eta)$, which from the definitions of $\Theta(j)$ and $\Theta^{\prime}(j+1, \eta)$ can be seen to be an element of $\Gamma_{k}(s(j)-1)$, and this shows $\mathbf{C}$. 


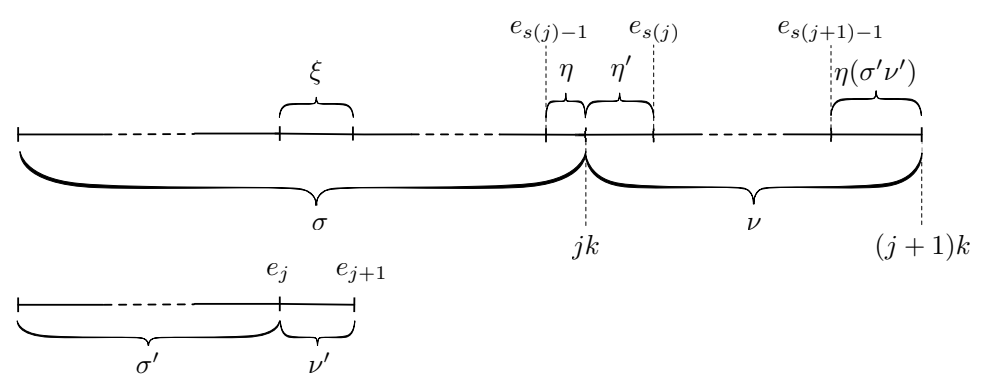

Fig. 3. A diagram depicting the construction of an element of $\mathcal{R}_{j+1}$

If $T^{j}(0)+\tau \notin \widetilde{F}$ then property $\mathbf{D}$ is trivial, so assume that $T^{j}(0)+\tau \in \widetilde{F}$. It is sufficient to show that if $\left(\sigma \nu_{1}, \sigma^{\prime} \nu_{1}^{\prime}\right),\left(\sigma \nu_{2}, \sigma^{\prime} \nu_{2}^{\prime}\right)$ are offspring of $\left(\sigma, \sigma^{\prime}\right) \in$ $\mathcal{R}_{j}$ then the projections of $Q\left(\sigma \nu_{1},\left.\sigma \nu_{1}\right|_{e_{j+1}}, \sigma^{\prime} \nu_{1}^{\prime}\right)$ and $Q\left(\sigma \nu_{2},\left.\sigma \nu_{2}\right|_{e_{j+1}}, \sigma^{\prime} \nu_{2}^{\prime}\right)$

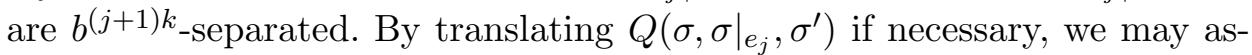
sume that $Q\left(\sigma,\left.\sigma\right|_{e_{j}}, \sigma^{\prime}\right)=\left[0, a^{-T^{j}(0)} b^{j k}\right] \times\left[0, b^{j k}\right]$, and therefore

$$
\begin{aligned}
\Pi_{\tau}\left(Q\left(\sigma \nu_{i},\left.\sigma \nu_{i}\right|_{e_{j+1}}, \sigma^{\prime} \nu_{i}^{\prime}\right)\right) & \left.=\left\{a^{-\tau} x+y:(x, y) \in Q\left(\sigma \nu_{i},\left.\sigma \nu_{i}\right|_{e_{j+1}}, \sigma^{\prime} \nu_{i}^{\prime}\right)\right)\right\} \\
& =b^{j k}\left\{a^{-\tau-T^{j}(0)} x+y:(x, y) \in Q\left(\nu_{i}, \xi, \nu_{i}^{\prime}\right)\right\} \\
& =b^{j k} \Pi_{\tau+T^{j}(0)} Q\left(\nu_{i}, \xi, \nu_{i}^{\prime}\right),
\end{aligned}
$$

where the fact that $\xi=\xi_{j}(\sigma)=\xi_{j}\left(\sigma \nu_{i}\right)$ was used on the penultimate line. By property (ii) of Corollary 3.2 , the family $\left\{\Pi_{\tau+T^{j}(0)}\left(Q\left(\nu_{i}, \xi, \nu_{i}^{\prime}\right)\right)\right\}$ is $b^{k}$ separated; it follows that the family

$$
\left\{\Pi_{\tau}\left(Q\left(\sigma \nu_{i},\left.\sigma \nu_{i}\right|_{e_{j+1}}, \sigma^{\prime} \nu_{i}^{\prime}\right)\right)\right\}
$$

is $b^{(j+1) k}$-separated, as desired. Property $\mathbf{E}$ follows from property (ii) of Corollary 3.2 and the fact that $\mathcal{R}_{j}$ satisfies property $\mathbf{C}$.

We can now complete the proof of Proposition 1.2 .

Proof of Proposition 1.2. By assumption $\log a / \log b$ is irrational, which guarantees the irrationality of $\alpha$. Thus, by Weyl's equidistribution theorem, the sequence $\left\{T^{j}(0)\right\}_{j}$ is equidistributed in $[0,1)$. Applying this to the set $F=\{x \in[0,1): \tau+x \in \widetilde{F}\}$ yields

$$
\lim _{j \rightarrow \infty} \frac{1}{j-j_{0}}\left|\left\{j_{0}<i \leq j: T^{i}(0) \in F\right\}\right|=\mathcal{L}^{1}(F) \geq 1-L \varepsilon^{1 / 2} .
$$

Let

$$
\Lambda_{\tau}=\bigcap_{j>j_{0}} \bigcup_{\left(\sigma, \sigma^{\prime}\right) \in \mathcal{R}_{j}} \Pi_{\tau}\left(Q\left(\sigma,\left.\sigma\right|_{e_{j}}, \sigma^{\prime}\right)\right),
$$

where $\mathcal{R}_{j}$ is the $j$ th level of the tree $\mathcal{R}$ guaranteed by Lemma 3.3. By property $\mathbf{A}$ this set is a countable intersection of nested compact sets, so 
$\Lambda_{\tau}$ is compact and non-empty. From the construction of $\Lambda_{\tau}$ it is clear that $\Lambda_{\tau} \subset \Pi_{\tau}(\Lambda)$.

We will estimate the dimension of $\Lambda_{\tau}$, and will do so in a standard way by means of constructing a natural measure supported on $\Lambda_{\tau}$. Let $\mu_{\tau}$ be the probability measure which assigns the same mass $\left|\mathcal{R}_{j}\right|^{-1}$ to each of the intervals $\Pi_{\tau}\left(Q\left(\sigma,\left.\sigma\right|_{e_{j}}, \sigma^{\prime}\right)\right)$ for $\left(\sigma, \sigma^{\prime}\right) \in \mathcal{R}_{j}$. This measure is well defined thanks to properties $\mathbf{D}$ and $\mathbf{E}$, and is supported on $\Lambda_{\tau}$. Let $x \in \Lambda_{\tau}$. Because of $\mathbf{D}$, for $j>j_{0}$ the interval $\left(x-b^{j k} / 2, x+b^{j k} / 2\right)$ intersects exactly one interval $\Pi_{\tau}\left(Q\left(\sigma,\left.\sigma\right|_{e_{j}}, \sigma^{\prime}\right)\right)$ with $\left(\sigma, \sigma^{\prime}\right) \in \mathcal{R}_{j}$, and thus

$$
\mu_{\tau}\left(x-b^{j k} / 2, x+b^{j k} / 2\right) \leq \mu_{\tau}\left(\Pi_{\tau}\left(Q\left(\sigma,\left.\sigma\right|_{e_{j}}, \sigma^{\prime}\right)\right)\right)=\left|\mathcal{R}_{j}\right|^{-1} .
$$

On the other hand, using $\mathbf{E}$ once again we obtain

$$
\log \left|\mathcal{R}_{j}\right| \geq\left|\left\{j_{0} \leq i<j: T^{i}(0) \in F\right\}\right| \log \left(c^{2} \delta^{\prime} \varepsilon b^{-k \gamma}\right) .
$$

Hence we learn from (3.9)-3.11) that if $j^{\prime}$ is large enough then for all $j>j^{\prime}$ we have

$$
\log \mu_{\tau}\left(x-b^{j k} / 2, x+b^{j k} / 2\right) \leq-\left(j-j_{0}\right)\left(1-2 L \varepsilon^{1 / 2}\right) \log \left(c^{2} \delta^{\prime} \varepsilon b^{-k \gamma}\right) .
$$

We then deduce that

$$
\begin{aligned}
\underline{\operatorname{dim}}_{\mathrm{loc}}\left(\mu_{\tau}, x\right) & :=\liminf _{j \rightarrow \infty} \frac{\log \left(\mu_{\tau}\left(x-b^{j k} / 2, x+b^{j k} / 2\right)\right)}{j k \log (b)-\log 2} \\
& \geq \liminf _{j \rightarrow \infty} \frac{\left(j-j_{0}\right)\left(1-2 L \varepsilon^{1 / 2}\right) \log \left(c^{2} \delta^{\prime} \varepsilon b^{-k \gamma}\right)}{\log 2-j k \log b} \\
& =\frac{\left(1-2 L \varepsilon^{1 / 2}\right) \log \left(c^{2} \delta^{\prime} \varepsilon b^{-k \gamma}\right)}{-k \log b}
\end{aligned}
$$

for any $x \in \Lambda_{\tau}$. From the Mass Distribution Principle (see e.g. [3, Proposition 2.3]) we conclude that

$$
\operatorname{dim}_{\mathrm{H}}\left(\Pi_{\tau}(\Lambda)\right) \geq \operatorname{dim}_{\mathrm{H}}\left(\Lambda_{\tau}\right) \geq \frac{\left(1-2 L \varepsilon^{1 / 2}\right) \log \left(c^{2} \delta^{\prime} \varepsilon b^{-k \gamma}\right)}{-k \log b} .
$$

The right hand side can be made arbitrarily close to $\gamma$ by letting $k \rightarrow \infty$ and then $\varepsilon \rightarrow 0$.

4. Proof of Theorem 1.1. To deduce Theorem 1.1 from Proposition 1.2 we show that any system satisfying the hypotheses of Theorem 1 can be arbitrarily well approximated from inside, in terms of dimension, by a homogeneous uniform fibre system. Moreover the matrix $A=\left(\begin{array}{ll}a & 0 \\ 0 & b\end{array}\right)$ in the homogeneous uniform fibre system needs to satisfy $\log a / \log b \notin \mathbb{Q}$. We start with two straightforward lemmas concerning when ratios of real numbers are rational.

LEMMA 4.1. If $x, y, r, t$ are non-zero real numbers and $\frac{x}{y}, \frac{x+r}{y+t}, \frac{x+2 r}{y+2 t} \in \mathbb{Q}$ then $\frac{r}{t} \in \mathbb{Q}$. 
Proof. This follows from straightforward calculations.

Lemma 4.2. Consider $\mathbb{R}$ as a vector space over $\mathbb{Q}$ and let $\left\{a_{1}, \ldots, a_{n}\right\} \subset$ $\mathbb{R}$ be linearly independent. If there exist $p_{1}, \ldots, p_{n}, q_{1}, \ldots q_{n} \in \mathbb{Q} \backslash\{0\}$ such that

$$
\frac{p_{1} a_{1}+\cdots+p_{n} a_{n}}{q_{1} a_{1}+\cdots+q_{n} a_{n}}=\frac{x}{y}
$$

where $x, y \in \mathbb{Z} \backslash\{0\}$ then $p_{i} / q_{i}=x / y$ for $1 \leq i \leq n$.

Proof. This follows by routine linear algebra.

We are now ready to prove the key lemma:

LEMma 4.3. Let $\left\{S_{i, j}\right\}$ be a Gatzouras-Lalley or Barański iterated function system and $\Lambda$ be the attractor. Given $\varepsilon>0$, there exists an iterated function system $\left\{A \cdot x+y_{l}\right\}_{l=1}^{N}$, where $A=\left(\begin{array}{ll}a & 0 \\ 0 & b\end{array}\right)$ is a diagonal matrix and the system has uniform horizontal fibres, such that the associated invariant set $\Lambda^{\prime}$ satisfies $\operatorname{dim}_{H}\left(\Lambda^{\prime}\right)>\operatorname{dim}_{H}(\Lambda)-\varepsilon$ and $\Lambda^{\prime} \subset \Lambda$. Moreover, if the iterated function system $\left\{S_{i, j}\right\}$ is of irrational type, we can choose $A$ such that $\log a / \log b \notin \mathbb{Q}$.

Proof. We just give the proof in the Gatzouras-Lalley case since the Barański case is proved in a similar way. Let $\mathbf{p}=\left(p_{1}, \ldots, p_{m}\right)$ be the unique probability vector for which the supremum in 1.1$)$ is attained, that is,

$$
\operatorname{dim}_{\mathrm{H}}(\Lambda)=\frac{\sum_{i=1}^{m} p_{i} \log p_{i}}{\sum_{i=1}^{m} p_{i} \log b_{i}}+t(\mathbf{p})
$$

where $t(\mathbf{p})$ is the unique real number satisfying

$$
\sum_{i=1}^{m} p_{i} \log \left(\sum_{j=1}^{n_{i}} a_{i j}^{t(\mathbf{p})}\right)=0 .
$$

For $(i, j) \in D=\left\{(i, j): 1 \leq i \leq m, 1 \leq j \leq n_{i}\right\}$ we let

$$
q_{i j}=\frac{p_{i} a_{i j}^{t(\mathbf{p})}}{\sum_{l=1}^{n_{i}} a_{i l}^{t(\mathbf{p})}} .
$$

For $k \in \mathbb{N}$, set $r(k)=\sum_{(i, j) \in D}\left\lceil k q_{i j}\right\rceil$, and let

$$
\Gamma_{k}:=\left\{\mathbf{d}=d_{1} \cdots d_{r(k)} \in D^{r(k)}:\left|\left\{1 \leq s \leq r(k): d_{s}=(i, j)\right\}\right|=\left\lceil k q_{i j}\right\rceil\right\},
$$

i.e. the set of all strings of length $r(k)$ over the alphabet $D$, for which the number of occurrences of the letter $(i, j)$ is equal to $\left\lceil k q_{i j}\right\rceil$. A simple combinatorial argument shows that

$$
\left|\Gamma_{k}\right|=\frac{r(k) !}{\prod_{(i, j) \in D}\left\lceil k q_{i j}\right\rceil !} .
$$

Consider the iterated function system $\left\{S_{d_{1}} \circ \cdots \circ S_{d_{r(k)}}\right\}_{\mathbf{d} \in \Gamma_{k}}$, and let its associated attractor be denoted by $\Lambda_{k}$. Clearly, $\Lambda_{k} \subset \Gamma$, and the linear 
part of each map has diagonal entries $\prod_{(i, j) \in D} a_{i j}^{\left\lceil k q_{i j}\right\rceil}, \prod_{(i, j) \in D} b_{i}^{\left\lceil k q_{i j}\right\rceil}$. Let $\pi: D \rightarrow\{1, \ldots, m\}$ denote the projection onto the second coordinate, and let

$$
\widetilde{\Gamma}_{k}=\left\{\pi\left(d_{1}\right) \cdots \pi\left(d_{r(k)}\right): d_{1} d_{2} \cdots d_{r(k)} \in \Gamma_{k}\right\},
$$

denote the projections of the strings in $\Gamma_{k}$ onto their second coordinates. Then we have

$$
\left|\widetilde{\Gamma}_{k}\right|=\frac{r(k) !}{\prod_{i=1}^{m}\left(\sum_{j=1}^{n_{i}}\left\lceil k q_{i j}\right\rceil\right) !} .
$$

For each $\sigma_{1} \cdots \sigma_{r(k)} \in \widetilde{\Gamma}_{k}$ the number of elements $d_{1} \cdots d_{r(k)} \in \Gamma_{k}$ such that $\pi\left(d_{i}\right)=\sigma_{i}$ for $i=1, \ldots, r(k)$ is equal to $\left|\Gamma_{k}\right| /\left|\widetilde{\Gamma}_{k}\right|$. Therefore, combining Stirling's formula with 4.1) and 4.2, we deduce

$$
\begin{aligned}
\operatorname{dim}_{\mathrm{H}}\left(\Lambda_{k}\right) & =\frac{\log \left|\widetilde{\Gamma_{k}}\right|}{-\sum_{(i, j) \in D}\left\lceil k q_{i j}\right\rceil \log b_{i}}+\frac{\log \left|\Gamma_{k}\right|-\log \left|\widetilde{\Gamma_{k}}\right|}{-\sum_{(i, j) \in D}\left\lceil k q_{i j}\right\rceil \log a_{i j}} \\
& =\frac{\sum_{i=1}^{m} p_{i} \log p_{i}}{\sum_{i=1}^{m} p_{i} \log b_{i}}+\frac{\sum_{i=1}^{m} p_{i} \log p_{i}-\sum_{(i, j) \in D} q_{i j} \log q_{i j}}{-\sum_{(i, j) \in D} q_{i j} \log a_{i j}}+o(1) \\
& =\frac{\sum_{i=1}^{m} p_{i} \log p_{i}}{\sum_{i=1}^{m} p_{i} \log b_{i}}+t(\mathbf{p})+o(1)=\operatorname{dim}_{\mathrm{H}}(\Lambda)+o(1) .
\end{aligned}
$$

This completes the proof of the first part of the lemma.

For the second part we will assume the system is of irrational type, and let

$$
\left\{f_{l}(x)=\left(\begin{array}{ll}
a & 0 \\
0 & b
\end{array}\right) x+y_{l}\right\}_{i=l}^{N}
$$

be the iterated function system from the first part with attractor $\Lambda_{k}$. If property (1) is satisfied then we can find a map $S_{i, j}$ in the original system with $\log a_{i j} / \log b_{i} \notin \mathbb{Q}$. By Lemma 4.1 one of

$$
\frac{\log a}{\log b}, \quad \frac{\log a+\log a_{i j}}{\log b+\log b_{i}}, \quad \frac{\log a+2 \log a_{i j}}{\log b+2 \log b_{i}}
$$

must be irrational. One of the three systems

$$
\begin{aligned}
& \left\{f_{l}(x)=\left(\begin{array}{ll}
a & 0 \\
0 & b
\end{array}\right) x+y_{l}\right\}_{l=1}^{N}, \\
& \left\{f_{l}(x)=S_{i, j} \circ\left(\left(\begin{array}{ll}
a & 0 \\
0 & b
\end{array}\right) x+y_{l}\right)\right\}_{l=1}^{N}, \\
& \left\{f_{l}(x)=S_{i, j}^{2} \circ\left(\left(\begin{array}{ll}
a & 0 \\
0 & b
\end{array}\right) x+y_{l}\right)\right\}_{l=1}^{N}
\end{aligned}
$$

will then be the required system. The difference between the dimension 
of the attractor of the new system and the dimension of $\Lambda_{k}$ can be made arbitrarily small by letting $k \rightarrow \infty$.

If property (2) is satisfied then we use Lemma 4.2. We can assume that $\log a_{i j} / \log b_{i}=c_{i j}^{-1} \in \mathbb{Q}$ for each $i, j$ and that $\log a / \log b=c^{-1} \in \mathbb{Q}$. By using property $(2)$ and considering $\mathbb{R}$ as a vector space over $\mathbb{Q}$ we can find $(k, l) \in D$ such that $\left\{\log a, \log a_{k l}\right\}$ is linearly independent. If

$$
\frac{\log a+\log a_{k l}}{\log b+\log b_{k}}=\frac{\log a+\log a_{k l}}{c \log a+c_{k l} \log a_{k l}} \in \mathbb{Q}
$$

then by Lemma 4.2, $c=c_{k l}$ and so $\log a / \log b=\log a_{k l} / \log b_{k}$ for any $(k, l) \in D$ where $\left\{\log a_{k l}, \log a\right\}$ is linearly independent. If all $\log a_{i j}$ are linearly independent with $\log a$ then this violates the second part of condition (2) and we can deduce that

$$
\frac{\log a+\log a_{i j}}{\log b+\log b_{i}} \notin \mathbb{Q}
$$

for some $(i, j) \in D$ and we can take our iterated function system as

$$
\left\{f_{l}(x)=S_{i, j} \circ\left(\left(\begin{array}{ll}
a & 0 \\
0 & b
\end{array}\right) x+y_{l}\right)\right\}_{l=1}^{N} .
$$

Finally if there also exists $(r, s) \in D$ where $\log a_{r s}=q \log a$ for some $q \in \mathbb{Q}$ then if

$$
\frac{\log a+\log a_{k l}+q \log a}{c \log a+c_{k l} \log a_{k l}+q c_{r s} \log a} \in \mathbb{Q}
$$

we can deduce that $c_{r}=c_{r s}$ since from the previous part we can assume that $c_{k l}=c$. For this to be true for all such $(r, s) \in D$ would violate condition $(2)$.

Using this lemma and then applying Proposition 1.2 completes the proof of Theorem 1.1. Note that while it is possible that in the Barański case we could have $a>b$ when we want to apply Proposition 1.2 , this is easily dealt with by swapping the $x$ and $y$ coordinates.

Acknowledgements. We thank Mark Pollicott for useful discussions. We would also like to thank the referees of the paper for their careful reading of the paper.

P.S. acknowledges support from EPSRC grant EP/E050441/1 and the University of Manchester.

\section{References}

[1] K. Barański, Hausdorff dimension of the limit sets of some planar geometric constructions, Adv. Math. 210 (2007), 215-245.

[2] T. Bedford, Crinkly curves, Markov partitions and box dimension in self-similar sets, $\mathrm{PhD}$ thesis, Univ. of Warwick, 1984. 
[3] K. Falconer, Techniques in Fractal Geometry, Wiley, Chichester, 1997.

[4] -, Fractal Geometry. Mathematical Foundations and Applications, 2nd ed., Wiley, Hoboken, NJ, 2003.

[5] M. Hochman and P. Shmerkin, Local entropy averages and projections of fractal measures, preprint, 2009; http://arxiv.org/abs/0910.1956.

[6] R. Kenyon and Y. Peres, Hausdorff dimensions of sofic affine-invariant sets, Israel J. Math. 94 (1996), 157-178.

[7] S. P. Lalley and D. Gatzouras, Hausdorff and box dimensions of certain self-affine fractals, Indiana Univ. Math. J. 41 (1992), 533-568.

[8] J. M. Marstrand, Some fundamental geometrical properties of plane sets of fractional dimensions, Proc. London Math. Soc. (3) (1954), 257-302.

[9] P. Mattila, Geometry of Sets and Measures in Euclidean Spaces, Cambridge Stud. Adv. Math. 44, Cambridge Univ. Press, Cambridge, 1995.

[10] C. McMullen, The Hausdorff dimension of general Sierpinski carpets, Nagoya Math. J. 96 (1984), 1-9.

[11] C. G. T. de A. Moreira, Sums of regular Cantor sets, dynamics and applications to number theory, Period. Math. Hungar. 37 (1998), 55-63.

[12] Y. Peres, The self-affine carpets of McMullen and Bedford have infinite Hausdorff measure, Math. Proc. Cambridge Philos. Soc. 116 (1994), 513-526.

[13] Y. Peres and W. Schlag, Smoothness of projections, Bernoulli convolutions, and the dimension of exceptions, Duke Math. J. 102 (2000), 193-251.

[14] Y. Peres and P. Shmerkin, Resonance between cantor sets, Ergodic Theory Dynam. Systems 29 (2009), 201-221.

Andrew Ferguson

Mathematics Institute

Zeeman Building

University of Warwick

Coventry CV4 7AL, UK

E-mail: a.j.ferguson@warwick.ac.uk

Pablo Shmerkin

School of Mathematics

and Centre for Interdisciplinary Computational

and Dynamical Analysis

Alan Turing Building

University of Manchester

Oxford Road, Manchester M13 9PL, UK

E-mail: Pablo.Shmerkin@manchester.ac.uk
Thomas Jordan Department of Mathematics University of Bristol University Walk, Clifton Bristol BS8 1TW, UK E-mail: Thomas.Jordan@bris.ac.uk

Received 12 March 2009;

in revised form 26 May 2010 